\title{
Alberti en la Argentina: los primeros pasos del exilio
}

\author{
Juana MARTíNEZ GÓMEZ \\ Departamento de Filología Española IV \\ Universidad Complutense de Madrid \\ juanamar@filol.ucm.es
}

\section{RESUMEN}

Este trabajo revisa minuciosamente los primeros meses del exilio argentino de Rafael Alberti, que transcurren no en Buenos Aires, donde después vivirá veintitrés años, sino en la provincia de Córdoba. Allí se instala recién llegado a la Argentina en una pequeña villa llamada El Totoral, donde empieza a tomar contacto con un nuevo paisaje y unos nuevos amigos que constituyen realmente su nueva patria. En ese refugio clandestino Alberti vuelve a la escritura, y sus primeros escritos argentinos traslucen la reacción del poeta ante ese nuevo entorno que le acoge lejos de España.

Palabras clave: Rafael Alberti, exilio español, Argentina.

\section{Alberti in Argentina: the first steps of exile}

\begin{abstract}
This work examines in detail the first months of the Argentinean exile of Rafael Alberti, that do not take place in Buenos Aires, where he will live during twenty three years afterwards, but in the province of Córdoba. The he will set his residence, just arrived to Argentina, in a little villa called El Totoral, where he begins to make contact with a new landscape and new friends that really become his new homeland. In that clandestine refuge Alberti goes back to writing and his first Argentinean texts show the reaction of the poet to this new environment that receives him away from Spain.
\end{abstract}

Key words: Rafael Alberti, Spanish exile, Argentina. 


\section{SOLOS Y SIN PATRIA}

Argentina se convierte en el lugar del exilio de Alberti de forma imprevista y casual. El 10 de febrero de 1940 se había embarcado en Marsella con destino a Chile con un pasaporte que le había proporcionado previamente Pablo Neruda en París. Su plan era desembarcar en Buenos Aires para seguir en un tren que lo llevara a Mendoza, y de ahí hasta la frontera con Chile, donde sería recibido por Neruda. Sin embargo, ese plan no pudo cumplirse porque, en la fecha prevista de su llegada a Chile, Neruda ya había abandonado su país para incorporarse al consulado chileno en México.

El matrimonio Alberti desembarcó en la ciudad porteña sin permiso para quedarse en Argentina pero, animados por los amigos que fueron a recibirlos al puerto, decidieron quedarse sin saber que permanecerían en este país durante veinticuatro años. En aquella época, en Argentina era difícil conseguir permiso de inmigración, y todavía durante muchos años más Rafael Alberti y María Teresa León no lograron obtener una autorización legal para residir en Buenos Aires, ni mucho menos un pasaporte para entrar y salir con normalidad (Soriano 1996: 153).

Los amigos les aconsejaron que solicitasen un permiso para visitar la ciudad de Buenos Aires durante cuatro días, tiempo que uno de esos amigos, Aráoz Alfaro, aprovechó para llevarlos a una vieja casona familiar en la villa de El Totoral, en la provincia de Córdoba, donde vivieron alrededor de un año de forma clandestina. Sólo cuando les llegó una cédula de identificación personal pudieron trasladarse a Buenos Aires.

En Argentina nunca vivieron una situación política cómoda, ya que entonces era un país contradictorio y dividido por el peronismo imperante, situación que se prolongó incluso después de la caída de Perón hasta extremos insoportables, que obligaron a la pareja a dejar la Argentina ${ }^{1}$. Lejos de la acogida generosa e impecable con que los exiliados españoles habían sido tratados por el gobierno mexicano de Lázaro Cárdenas, en Argentina los Alberti se encontraron con numerosos obstáculos y, en ocasiones, con serias dificultades que el mismo poeta relata:

Durante el plan Conintes teníamos intervenido el teléfono, nos revisaban la correspondencia y estábamos en una lista negra. Todo se nos fue estrechando cada vez más, incluida nuestra situación económica. Cuando llegó Frondizi la cosa se nos puso peor. Una noche tres tipos allanaron mi casa, estando mi señora sola. Venían a detenerme pistola en mano. Detuvieron a Miguel Ángel Asturias y a mí no me agarraron porque había salido. Tuve que esconderme en Castelar durante

\footnotetext{
1 “[...] después de diecinueve años sin pasaporte, durante los cuales sólo pude viajar de Argentina a Uruguay, bien en avión, no más de media hora, o en un barco que atravesaba el río de la Plata durante la noche, dejándonos, algo pasado el amanecer, en el puerto de Montevideo. Pero un día alguien me comunicó que había aparecido la noticia de que el consulado franquista concedía pasaporte a los exiliados, pero únicamente a aquellos españoles 'que no tuviesen las manos manchadas de sangre' [...] iqué maravilla poder salir a respirar después de tantos años forzosamente prisionero, paralizado en el Río de la Plata, en la República Argentina, amada de verdad pero cada vez más estrecha y preocupante después del peronismo, de aquellos cohibidos gobiernos democráticos". (Alberti 1997, 2: 202-203)
} 
un mes. Cuando pude salir, dije basta. El 28 de mayo de 1964 salimos de la Argentina con gran dolor. Hacia veinticuatro años que estábamos allí, pero un día se nos hizo imposible. (Soriano 1996: 154-155)

En tales circunstancias, mientras que Alberti mantenía su compromiso ideológico con España, en lo que respecta a la Argentina, sin embargo, prefirió mantenerse al margen de los problemas políticos locales para cifrar su compromiso con el hombre y la estética, porque, aunque para los gobiernos argentinos era un antifranquista tachado continuamente de "rojo" por la prensa, él se sintió respaldado desde el principio por el pueblo argentino, que enseguida le mostró su ayuda y una gran simpatía por la causa española.

Mucho se ha hablado del desarraigo y la soledad de Alberti, e incluso de su falta de solidaridad con el ambiente social, es decir, del aislamiento de la realidad inmediata por un estado de permanente nostalgia de España; pero no hay que olvidar que la atmósfera política en Argentina no era la más propicia para el diálogo y la integración, y Alberti no encontró ningún aliciente para desarrollar alguna clase de actividad en este terreno. Su inadaptación al medio no solo tiene que ver con la nostalgia de España sino con la incompatibilidad ideológica entre el poeta y los gobiernos argentinos con los que le tocó vivir.

En este estado de cosas cabe preguntarse: ¿Cómo se produce el primer contacto con el mundo argentino? ¿Cómo reacciona el poeta? ¿Cómo es su relación con el nuevo entorno? Preguntas todas cuya respuesta, que trataremos de dar aquí, determina los primeros pasos de Alberti en el exilio y su posterior grado de integración en Argentina.

Cuando María Teresa León, compañera inseparable de Alberti en la experiencia del exilio, narra en sus memorias el arribo a la capital argentina, fija en la letra las primeras emociones e inquietudes que cargan de sentido los pasos iniciales del exilio en Argentina: "[...] desembarcamos nosotros en el puerto de Buenos Aires muy cargados de penas. ¿Qué haríamos solos y sin patria?" (León 1999: 508). Pero también agrega de inmediato: "Nuestra patria iban a ser desde ese momento en adelante nuestros amigos". Patria es el término esencial, que repite para asociarlo al de amistad y, al mismo tiempo que patria y amistad se identifican, la patria se distancia de la noción de tierra, lugar o geografía.

En los primeros años del exilio, cuando la tierra y la patria iniciales están recién perdidas, la vivencia de la nueva tierra está muy lejos de coincidir con la de la nueva patria que se les brinda. Cuando María Zambrano reflexiona sobre el exilio años después de su experiencia, también establece diferencias entre los conceptos de patria y tierra; para ella, "la patria es una categoría histórica" a la que otorga un contenido mucho más amplio que el de lugar: "La patria es lugar de historia, tierra donde una historia fue sembrada un día" (Zambrano 2004: 42). Mientras que la geografía, la tierra, es lo desconocido, lo que va a hacer que el poeta se sienta extraño y ajeno y, en consecuencia, ahonde en su pena y en su sensación de lejanía; la patria que le ofrecen los amigos, la amistad, refuerza lo propio, lo íntimo y privado, pues ellos acogen a Alberti y le descargan de sus tristezas, para contribuir a "sembrar" y afianzar las raíces de su nuevo "lugar de historia". 
De momento hablaremos de esta amistad como nueva patria de Alberti, para hacerlo más tarde sobre la tierra. La amistad se convierte en el motor que impulsa al poeta español a superar el estado emocional derivado de la soledad y la pena con los que llega a Buenos Aires, y constituyen lo que él llama las primeras "redes de esperanza". Cuando recuerda este momento en sus memorias dice: "Y al fin, América, Buenos Aires, la Argentina, de tránsito para Chile. ¿Para Chile? No, porque me quedo en Buenos Aires donde buenas manos amigas me tienden redes de esperanza" (Alberti 1997, 1: 83).

\section{REDES DE ESPERANZA}

Las primeras experiencias del exilio de Alberti en Argentina, sin embargo, no transcurren en Buenos Aires sino en la provincia de Córdoba, cuya capital es la segunda ciudad en importancia del país. El poeta llegó al puerto argentino el 3 de marzo de 1940 y de inmediato viajó hacia el norte para instalarse en la casa del amigo $^{2}$, en la villa de El Totoral, durante casi un año.

El papel de la amistad es fundamental en este otro enclave inesperado del exilio y está relacionado con dos amigos muy especiales. El primero de ellos es Rodolfo Aráoz Alfaro, uno de los muchos amigos que acudieron a recibirlo el día de su llegada al puerto de Buenos Aires, y al que distingue con el paso del tiempo en su memoria como "gentilísimo amigo y camarada". Era entonces un prestigioso abogado, amigo también de Neruda y destacado miembro del Partido Comunista, y ocupa un papel crucial en la vida argentina de Alberti, entre otras cosas, porque le acoge y le proporciona un refugio en la provincia de Córdoba para que pase desapercibido los primeros meses de su estancia, antes de legalizar su situación e instalarse en Buenos Aires. Casi treinta años después, Alberti recordaba este primer contacto con el mundo argentino: "Nos retuvo el campo de Córdoba, un pueblo de totoras -o cañas- como flechas, El Totoral, con su río pequeño, quien nos retuvo casi un año junto a las alamedas de un grande y nuevo amigo, Rodolfo Aráoz Alfaro" (Alberti 1969: 11).

El otro amigo fundamental en estos primeros momentos es Deodoro Roca, a quien conoció ya en Córdoba, y en seguida lo consideró como "una nueva y grande amistad". Deodoro Roca era también abogado y se había convertido en uno de los

2 "La casa [...] fue comprada a principios del siglo por mi padre, con el propósito de echarla abajo y construir una nueva. Mi madre se enamoró de sus anchas paredes, de piedra y adobe, fresca en verano y con tibio abrigo en invierno, y resolvió modernizarla. [...] Cuando fue mía, traté de restituirle su estilo original, arrancando en lo posible todo lo extraño y agregado, y añadiéndole grandes galerías y hornacinas. [...] Mi casa se llamaba "El Kremlin". Es decir, así la llamaban mis enemigos de Córdoba. Siempre estuvo llena de aborrecidos izquierdistas o intelectuales que podían haberlo sido o pasaban por tales. Tristan Maroff, los Alberti -emigrados de la guerra española-, Victor Delhes, maravilloso artesano flamenco del grabado en madera, Deodoro Roca, Raúl González Tuñón y Amparo Mom, los Jorge -Faustino y Sarita-, Mario Bravo, Rodolfo Ghioldi, Toño Salazar y Carmela, su mujer, Pablo Neruda, Córdova Iturburu y su mujer Carmen de la Serna, y hasta parece que estuvo varias veces en su juventud el Che Guevara. Ahora llegan chilenos de todas clases y reyes del folklore: Cafrune, Tejada Gómez, Mercedes Sosa, o astros de la literatura como Sábato". (Aráoz Alfaro 1967: 68-71) 
escritores políticos más importantes de su tiempo. Había sido el autor intelectual de la Reforma Universitaria de 1918, verdadera revolución de la organización universitaria respecto de los principios que la habían sustentado hasta entonces. Con su intervención, la transformación universitaria se inició en Córdoba, y se extendió por el resto de Argentina y otros países latinoamericanos. Alberti conectó rápidamente con él por su condición iconoclasta y su pasión por la poesía.

Deodoro Roca le ayudó a normalizar su vida intelectual introduciéndolo en los medios culturales y en la prensa cordobesa, que, por su parte, hizo un seguimiento minucioso de las actividades del poeta español. El diario La voz del interior, especialmente, mantuvo muy bien informados a sus lectores de cuanto recital o conferencia ofreciese el poeta español. A través de este diario conocemos la gran expectación que despertaron las conferencias que Alberti ofreció los días 4 y 6 de junio de 1940 en el Teatro Rivera Indarte y en la Universidad, respectivamente.

La primera conferencia, que fue auspiciada por el Círculo de la Prensa de Córdoba y considerada como "un verdadero acontecimiento en la vida intelectual" de la ciudad, fue anunciada como "un honor y como una verdadera primicia" porque ponía al público cordobés "en contacto con tan eximia figura de la lírica hispánica" (La voz del interior, Córdoba, 3 de junio de 1940). La conferencia versó sobre "García Lorca, poeta y amigo" y fue presentada en el Teatro Rivera Indarte por el propio Deodoro Roca con palabras que, además de mostrar el gran conocimiento que tenía de España, no escatimaban la admiración que el abogado cordobés sentía por el poeta español. Entonces lo llamó "gran poeta arisco y magnífico, purificador (también) de la poesía y sus técnicas, jefe de un pelotón de fusileros de las retóricas", y tomó en consideración la poesía de Alberti como parte de su tierra, "como islotes flotantes de una España hundida" (Roca 1972: 115-116).

Alberti volvió a sentir la proximidad del pueblo cordobés dos días más tarde, en otra conferencia que tuvo lugar en el Salón de Actos de la Facultad de Derecho de la Universidad de Córdoba, una sesión multitudinaria en la que, junto al Rector, el cuadro de profesores y los alumnos, el poeta convocó a muchos otros asistentes. Fue presentado por uno de los profesores, quien en su discurso quiso transmitirle muy efusivamente el afecto y la admiración que todos sentían hacia él. Alberti disertó sobre "Una generación de poetas españoles" que, según la nota de prensa aparecida, "desarrolló magistralmente, matizándolo con el recitado de numerosos poemas" (La voz del interior, Córdoba, 7 de junio de 1940).

Cuando murió Deodoro Roca dos años más tarde, Alberti, que se atribuyó el título de "su último y más reciente amigo", recordaba emocionado sus palabras de presentación en el teatro de Córdoba:

No olvidaré nunca las palabras de hondo conocimiento y amor, breve reflejo de su clara conducta durante nuestra guerra, que puso como prólogo a mi conferencia sobre Federico García Lorca en el teatro Rivera Indarte de la Córdoba de sus batallas. No, no solo yo, sino los españoles todos de este impuesto peregrinaje por tierras de América, le olvidaremos, le perderemos de nuestra memoria. (Roca 1972: 148-149) 
Junto a estos amigos y al afecto que pudo percibir en la ciudadanía cordobesa, otro amigo que le proporcionó una ayuda no menos estimable que la de los anteriores fue Gonzalo Losada, "nuestro grande y generoso Gonzalo Losada, un nuevo editor lleno de genio e iniciativas". También se encontraba él entre los amigos que lo esperaban en el puerto de Buenos Aires, y desde allí le tendió de inmediato otras buenas redes de esperanza, pues le adelantó el dinero del que iba a ser su próximo libro, Entre el clavel y la espada, cuyos poemas había comenzado a escribir en Francia antes de partir, y esto le permitió resolver económicamente la supervivencia inmediata ${ }^{3}$. No es preciso insistir en lo que significó posteriormente la editorial Losada para la difusión de la obra del poeta español, pero de momento su casa editorial le avalaba y publicaba el primer libro de poemas de Alberti terminado en Argentina.

La fuerza de estas amistades fue tan grande que se convirtió en un acicate para el Alberti creador. Aunque él no había interrumpido su creación poética desde Francia hasta Argentina - pues mientras navegaba por alta mar siguió componiendo poemas-, repite varias veces que en Córdoba "crece de nuevo a la poesía", es decir, se reaviva, evoluciona, se enriquece: "Crecí de nuevo a la poesía entre los álamos y los sauces que circundaban la vieja casona del amigo. Árboles serios y profundos, muy de Antonio Machado, me llevaban constantemente a su recuerdo" (Alberti 1969: 11).

Alberti no solo se animó a escribir sino que también volvió a recitar rodeado del calor del público. Tres meses después de su llegada, el 28 de junio, tuvo lugar en el Museo Histórico Provincial, antigua casa del Virrey Sobremonte, un sonado recital poético en el que fue presentado por la escritora Sara de Maza, quien de manera rigurosa destacó los aspectos más sobresalientes de la obra del poeta. Alberti, estimulado por esos nuevos amigos anónimos, no solo recitó muchos de sus poemas y explicó los nuevos derroteros de su poesía, sino que también ofreció como primicia la primera lectura de algunos de los poemas de su nuevo libro, Entre el clavel y la espada, que estaba terminando "en su amable retiro de Totoral, frente al bello paisaje cordobés". El poeta quiso hacer entender a los asistentes el profundo sentido político de ese libro porque "entre el clavel y la espada era como vivían muchos españoles que andaban por los caminos del mundo, esa espada -agregó- que pende sobre todos nosotros y que los que hemos vivido en Europa hemos sentido tan cerca de nuestras mejillas" (La voz del interior, Córdoba, 29 de junio de 1940).

Cuando veinticuatro años después de su llegada tiene que despedirse de Argentina, no puede olvidar la impronta de Córdoba, de sus amigos y de su paisaje en su impulso creador, e insiste: "Crecí de nuevo a la poesía entre esos álamos. La voz de Deodoro Roca me presentó en mi primera conferencia con un largo discurso del que

3 "Nos pagó durante varios meses los derechos del libro, como también el resto que me debía por mi Antología poética, publicada unos meses antes. Gonzalo Losada era una alto empleado de Espasa-Calpe, que se desgajó de la gran editorial, cuando nuestra guerra, por ser republicano y no del otro lado como era la gran casa española. Editor nuevo, audaz, publicó por primera vez la obra de tantos poetas y escritores latinoamericanos que antes nadie se había atrevido con ellos" (Alberti 1997, 1: 135). Emilia Zuleta explica que Losada residía en Buenos Aires desde 1928 como gerente de Espasa-Calpe Argentina. En 1938 funda la editorial Losada con Guillermo de Torre y Atilio Rossi. Les acompañarán también en la empresa Pedro Henríquez Ureña, Francisco Romero y Amado Alonso (Zuleta 1999). 
dijo graciosamente que no había tenido tiempo de hacerlo más corto. Y con su espaldarazo eché a andar" (Alberti 1978: 615).

\section{ENTRE LOS ÁLAMOS Y LOS SAUCES DE LA AMÉRICA AUSTRAL}

En efecto, en Córdoba echó a andar de nuevo la palabra creadora de Alberti. Sus primeros pasos argentinos transcurren, pues, junto a sus amigos entre los álamos y los sauces cordobeses, entre "los árboles serios y profundos" del Totoral ${ }^{4}$, y junto a estos árboles se componen sus primeros renglones y sus primeros versos escritos en Argentina. La naturaleza y la amistad cordobesas son las primeras que cobijan y alimentan literal y literariamente a Alberti en su reciente exilio.

En El Totoral compone su primer texto en prosa escrito en Argentina, "Entre los álamos argentinos", una prosa poética en la que narra su primer contacto con una naturaleza nueva. Su descubrimiento de la fauna y la flora del lugar incorpora a su literatura voces desconocidas que se mezclan con las antiguas y familiares.

En aquellos campos del Totoral argentino, entrelazándose los hilos de sangre que traían los vientos que llegaban de Europa, había el pitido de los benteveos, la charla estridente de los loros, el zureo de las palomas, junto a caballos que corrían hacia los montes azulados y el estruendo homérico de las vacas y toros en el baño de las haciendas. (Alberti 1969: 13)

Si el calor de la amistad le iba proporcionando a Alberti una nueva patria, él no acababa de identificarse con la tierra que pisaba. Comienza aquí a manifestarse de inmediato su extrañeza, su sentimiento de ser un extraño, un extranjero. Aunque se pasea a gusto por las callecitas del pueblo, bajo los árboles, entre los pájaros y las yerbas aromáticas del campo, que enseguida se le hacen familiares, su existencia es amarga e inestable, pues vive todavía con la esperanza de volver pronto a la "patria perdida"; en ese nuevo entorno no se siente seguro ni tranquilo, con el convencimiento de estar de paso, con la sensación a flor de piel de ser un "español errante", pero con la esperanza de un regreso no muy lejano.

En su primer escrito cordobés, si bien los árboles de El Totoral le sirven de consuelo, presta gran atención a aquellos álamos que se mueren secos, derribados por los vendavales del verano, porque tienen algo de "cosa humana" por su "larga quejumbre", "que se descuaja de la profunda raíz terrena". Él, hombre descuajado también, parece identificarse con esa imagen de los árboles abandonados y "muertos de sed", que han sido arrancados de la tierra como queriendo decir que a ambos, árboles y poeta, se les vuelve difícil o imposible la existencia sin la materia primordial nutricia.

\footnotetext{
${ }^{4}$ El Totoral, "nombre delicioso, que figura en las memoria de la época, pues los ejércitos se detenían siempre a su vera, arbolada y con un arroyo de aguas claras, en tiempos en que el norte de Córdoba debía ser sólo un monte cerrado de algarrobos, talas y barbas de tigre. [...] Totoral es un pueblo antiguo, con su arroyo siempre abundante, algunas casa viejas y hasta jesuitas - como la merced de Jerónimo Luis de Cabrera, fundador de Córdoba, que se llama San Antonio de la Buena Vista”. (Aráoz Alfaro 1967: 68-69)
} 
Los álamos y los sauces de El Totoral proporcionan a Alberti un componente esencial para la quinta parte de su libro Entre el clavel y la espada, a la que titula "De los álamos y los sauces. En recuerdo de Antonio Machado". Los árboles adquieren una doble connotación, pues al lado de los árboles descuajados y secos hay otros árboles que representan la vida, la patria, la amistad, y con ellos dialoga y se consuela.

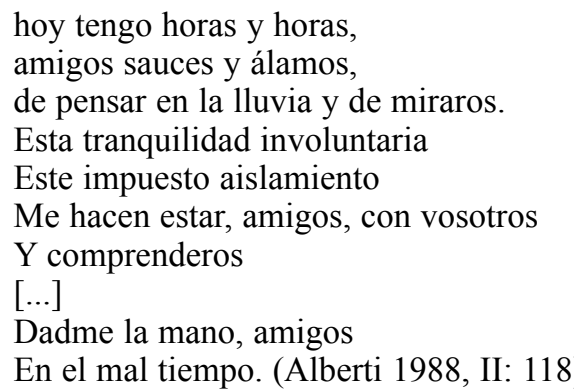

Igualmente, los árboles son un espejo de su propia realidad que le hacen caer en la cuenta del mundo quebrado en el que vive, un mundo escindido entre un aquí y un allí y entre un antes y un después. Unos algarrobos le sirven para resaltar esa dualidad que comporta su "vida rota" en el exilio.

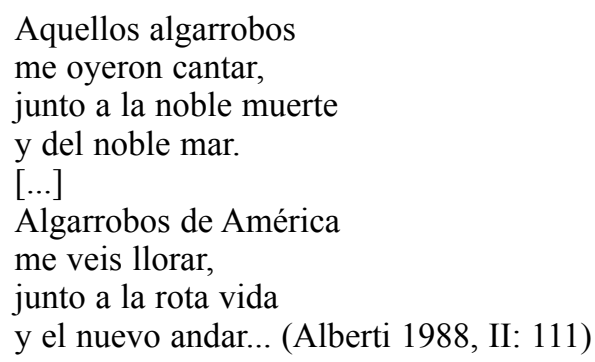

Estos binomios marcan todo el libro Entre el clavel y la espada, que se desliza entre el consuelo dulce del clavel y la angustia sangrante de la espada, los dos polos entre los que se desenvuelven las emociones del poeta, bandeando entre sentimientos opuestos, que están determinados también por dos espacios y dos tiempos diferentes, como él mismo indica en el título del prólogo, "De ayer para hoy". En él expresa con claridad la nueva situación en la que vive, "hincado" entre una flor y una espada: "de un lado, un seco olor a sangre pisoteada; de otro, un aroma a jardines, a amanecer diario, a vida fresca, fuerte, inexpugnable" (Alberti 1988, II: 61).

A partir de la tercera parte del libro, titulada "Metamorfosis del clavel", se hace patente la existencia de una tierra distinta y empiezan las manifestaciones de una nueva experiencia con el espacio adquirida tras el exilio; manifestaciones que se incrementan en la cuarta, titulada "Toro en el mar (Elegía sobre un mapa perdido)". Aquí el nombre de España no se pronuncia sino que se la menciona con connota- 
ciones de lejanía como "aquel país", que se reconoce por su mapa con forma de piel de toro. El tono elegiaco determina los versos de esta parte que siempre sitúa al toro en el otro lado del mar, separado del poeta por una inmensa distancia respecto del lugar donde se encuentra: "Te oigo mugir en medio de la noche / por encima del mar, también bramando" (Alberti 1988, II: 107).

Ese nuevo lugar que él habita, distante del toro, tampoco se nombra como Argentina sino de manera amplia América o Hemisferio Austral, y suele configurarse como una noche carente de líneas claras y definidas; es decir, esa nueva tierra es un espacio invisible, oscuro, ausente de contornos al que el poeta se refiere como "noche siempre" o "planetaria noche triste", desde la que recuerda España y le habla.

Quiero decirte, toro, que en América, desde donde en ti pienso -noche siempre-, se presencian los mapas, esos grandes deshabitados sueños que es la Tierra. [...] Yo quería dormir tranquilo, un poco, pues me hace falta, como a tí; quería, cuan largo y triste como tú, tumbarme siquiera en el retraso de una aurora.

Pero me he levantado, ya que andaba, párpado insomne el fijo pensamiento, pensando en ti, para -iluceros sordos en la noche de América!- decírtelo. (Alberti 1988, II: 109)

Estos poemas iniciales del exilio muestran un fuerte sentimiento de destierro, de pérdida de la tierra y, al tiempo, un escaso interés por el suelo que pisa, donde se halla intranquilo, inseguro y muy desorientado. Se siente como su paloma, la paloma que se equivocaba, cuando "por ir al norte fue al sur", porque desconoce los cielos y las estrellas.

Se puede decir que Alberti, aparte de ser un desterrado, en Córdoba es también, si se me permite el neologismo, un "descielado", que no reconoce el cielo que le cubre:

Busco, nostálgico, constelaciones que no encuentro, que yo sé que no están, estrellas familiares, que se quedaron por el otro, esperándome. ¿Dónde andará aquella Osa Mayor que se iba abriendo, grande, con el girar de las horas, hasta correr, hacia la madrugada, en un ancho galope sobre los picos estivales del Guadarrama? La Cruz del Sur, más luminosa aún junto al profundo Saco de Carbón de la Vía Láctea, me mira recordándome mi suerte. No ando, no, bajo las alamedas, las castellanas choperas de Antonio Machado. (Alberti 1978: 561-562)

El cielo y la tierra ajena parecen confabularse contra él para desorientarlo. No cabe duda de que vivir en el hemisferio sur parece convertirse en otra dificultad añadida para Alberti respecto a los exiliados españoles en México, que no cambiaron de 
hemisferio ${ }^{5}$. El poeta lo vive al principio como un desorden, como un elemento inquietante que se agrega a sus desdichas, aunque con el tiempo acabará acostumbrándose. Es muy sintomático que, muchos años después, de vuelta ya en Madrid, sea el recuerdo de la noche austral la que señale el cambio tan profundo que se ha producido en su vida lejos ya del exilio:

Noche. Cuando desde el balcón al Guadarrama en que estoy, miro al cielo buscando la Osa Mayor, que se va abriendo, tendida sobre los montes, me emerge, de un agujero negro de la Vía Láctea, la geometría perfecta de la Cruz del Sur, recuerdo entonces que mi vida corrió, hace ya muchos años, bajo la noche austral de América, lejos, muy lejos de estos cielos españoles, que puedo ahora contemplar más tranquilo. (Alberti 1997, 2: 163)

\section{BIBLIOGRAFÍA}

ALBERTI, Rafael (1969): Un poeta español en el Río de la Plata. Roma: Istituto Italo-latinoamericano.

- (1978): El poeta en la calle (Obra Civil). Edición de Aitana Alberti. Madrid: Aguilar.

- (1988): Obras completas II y III. Poesía (1939-1963) y (1964-1988). Edición de Luis García Montero. Madrid: Aguilar.

- (1997): La arboleda perdida, 1 y 2. Madrid: Alianza Editorial.

ARÁOZ ALFARO, Rodolfo (1967): El recuerdo y las cárceles (Memorias amables). Buenos Aires: Ediciones de la Flor.

ROCA, Deodoro (1972): Prohibido prohibir. Buenos Aires: La Bastilla.

SORIANO, Osvaldo (1996): Piratas, fantasmas y dinosaurios. Bogotá: Grupo Editorial Norma.

ZULETA, Emilia (1999): Españoles en la Argentina. El exilio literario de 1936. Buenos Aires: Ediciones Atril.

\footnotetext{
${ }^{5}$ En Córdoba también se producen otras alteraciones cotidianas derivadas de su ubicación en otro hemisferio, como cuando cumple los 39 años un 16 de diciembre: "Pero allí era verano. Muy lejos del ambiente navideño de Europa. Se encendía en el viejo salón de la quinta de Aráoz Alfaro el árbol de Noel, colgado de regalos y visitado a toda velocidad, de cuando en cuando, por los relampagueantes colibríes que penetraban por las puertas en busca de su nido colgado de alguna cuerda que pendía de la rama de un árbol seco en el jardín" (Alberti 1997, 2: 390).
} 